\title{
Produção científica sobre o acolhimento nos serviços de saúde na perspectiva cienciométrica
}

\author{
Scientific production on welcoming health services from a cenciometric perspective \\ Producción científica sobre la acogida de servicios de salud desde una perspectiva \\ cenciométrica
}

Ana Luiza Montalvão Seixas ${ }^{1}$, Valéria Gonzaga Botelho de Oliveira ${ }^{2}$, Virginia Ruas Santos ${ }^{3}$, Ana Cecília Dias Batista ${ }^{4}$, Giuliana de Fátima Gonçalves Braga ${ }^{3}$, Ravena Amaral da Conceição ${ }^{5}$, Karla Talita Santos Silva ${ }^{3}$, Sylmara Corrêa Monteiro ${ }^{6}$, Bruno de Pinho Amaral ${ }^{5}$, Renê Ferreira da Silva Junior $^{7 *}$.

\section{RESUMO}

Objetivo: Avaliar o nível de conhecimento e sistematização da produção científica vinculada à Política Nacional de Humanização em sua diretriz de acolhimento. Métodos: Realizou-se um estudo cienciométrico entre os meses de setembro e outubro de 2020, tendo como descritor o termo acolhimento nas bases de dados secundários United States National Library of Medicine e na Scientific Eletronic Library Online. Resultados: Foram selecionados 67 artigos sobre acolhimento, o ano de 2014 teve o maior número de publicações, o maior quantitativo de estudos tiveram como foco a Unidade Básica de Saúde e setores hospitalares, seguido de estudos que abordavam o acolhimento no Centro de Atenção Psicossocial, quanto ao local de origem São Paulo, Ceará, Pernambuco e Minas Gerais obtiveram 49\% dos 67 artigos relacionados ao acolhimento nos serviços de saúde, $47,7 \%$ dos artigos não foram classificados nos estratos qualis, sendo A2 com 11,9\% e B1 com 32,8\%. Considerações finais: Após dezessete anos da implementação da Política Nacional de Humanização em relação a sua diretriz acolhimento, constou-se que houve produções voltadas mais fortemente à implementação do acolhimento, sobretudo, no cenário da Atenção Primária à Saúde, nesse sentido, houve uma produção limitada em relação às práticas de acolhimento como determina a política.

Palavras-chave: Acolhimento, Humanização da assistência, Política pública.

\begin{abstract}
Objective: To assess the level of knowledge and systematization of scientific production linked to the National Humanization Policy in its welcoming guideline. Methods: A scientometric study was carried out between the months of September and October 2020, using the term host in the secondary databases United States National Library of Medicine and Scientific Electronic Library Online as its descriptor. Results: 67 articles on reception were selected, the year 2014 had the largest number of publications, the largest number of studies focused on the Basic Health Unit and hospital sectors, followed by studies that addressed the reception at the Psychosocial Care Center, as to the place of origin São Paulo, Ceará, Pernambuco and Minas Gerais obtained $49 \%$ of the 67 articles related to the reception in health services, $47.7 \%$ of the articles were not classified in the qualitative strata, being A2 with $11.9 \%$ and B1 with $32.8 \%$. Final considerations: Seventeen years after the implementation of the National Humanization Policy in relation to its welcoming directive, it was found that there were productions more strongly focused on the implementation of welcoming, especially in the Primary Health Care scenario, in this sense, there was limited production in relation to reception practices as determined by the policy.
\end{abstract}

Key words: User embracement, Humanization of assistance, Public policy.

\footnotetext{
${ }^{1}$ Faculdade de Saúde Ibituruna (FASI), Montes Claros - MG.

${ }^{2}$ Instituto Federal de Educação, Ciência e Tecnologia do Norte de Minas Gerais (IFNMG), Almenara - MG.

${ }^{3}$ Faculdades Unidas do Norte de Minas Gerais (FUNORTE), Montes Claros - MG.

${ }^{4}$ Universidade Federal de Mato Grosso (UFMT), Cuiabá - MG.

5 Universidade Estadual de Montes Claros (UNIMONTES), Montes Claros - MG.

${ }^{6}$ Instituto Federal de Ciência, Educação e Tecnologia do Norte de Minas Gerais (IFNMG), Montes Claros - MG.

${ }^{7}$ Instituto Federal de Educação, Ciência e Tecnologia de Santa Catarina (IFSC), Joinville - SC.

*E-mail: renejunior_deny@hotmail.com
} 


\section{RESUMEN}

Objetivo: Evaluar el nivel de conocimiento y sistematización de la producción científica vinculada a la Política Nacional de Humanización en su directriz de acogida. Métodos: Se realizó un estudio cienciométrico entre los meses de septiembre y octubre de 2020, utilizando como descriptor el término host en las bases de datos secundarias Biblioteca Nacional de Medicina de Estados Unidos y Biblioteca Electrónica Científica en Línea. Resultados: Se seleccionaron 67 artículos sobre recepción, el año 2014 tuvo el mayor número de publicaciones, el mayor número de estudios enfocados a la Unidad Básica de Salud y sectores hospitalarios, seguido de estudios que abordaron la recepción en el Centro de Atención Psicosocial, en cuanto al lugar de origen São Paulo, Ceará, Pernambuco y Minas Gerais obtuvieron el $49 \%$ de los 67 artículos relacionados con la recepción en los servicios de salud, el $47,7 \%$ de los artículos no fueron clasificados en el estrato cualitativo, siendo A2 con $11,9 \%$ y B1 con 32,8\%. Consideraciones finales: Diecisiete años después de la implementación de la Política Nacional de Humanización en relación a su directriz de acogida, se constató que existían producciones más fuertemente enfocadas a la implementación de acogida, especialmente en el ámbito de la Atención Primaria de Salud, en este sentido, hubo producción limitada en relación con las prácticas de recepción determinadas por la política.

Palabras clave: Acogimiento, Humanización de la atención, Política pública.

\section{INTRODUÇÃO}

A Política Nacional de Humanização (PNH) foi lançada em 2003 pelo Ministério da Saúde visando ao fortalecimento do Sistema Único de Saúde (SUS) para inovação e mudanças na saúde para intervir e pensar no cotidiano da saúde pública, assegurando por meio dos direitos de todo cidadão acesso ao serviço com aspecto igualitário e universal (MARTINS CP e LUZIO CA, 2017). A humanização não deve ser limitada somente a um espaço físico e com conjunto de normas que devem ser cumpridas, considerando-se que será no encontro entre pessoas, teorias e técnicas que haverá uma produção de saúde (MARTINS CP, 2015).

$\mathrm{O}$ acolhimento é uma das diretrizes da $\mathrm{PNH}$, sendo assim o acolhimento e a humanização caminham juntas para a melhoria do atendimento, é importante que exista esse vínculo entre eles, um dos grandes desafios da humanização é a implantação do acolhimento pela equipe multiprofissional (NOBRE JPS, et al., 2016). Acolher refere-se a escutar de maneira adequada e qualificada as necessidades dos usuários, possibilitando uma conduta positiva e responsabilizando-se pela redução ou eliminação dos problemas ou sofrimentos apresentados pelos usuários (BORGES JDM e SILVA LAA, 2015).

O acolhimento é uma das ferramentas que proporciona vincular profissionais e usuários, sendo assim é importante que haja uma reflexão de como funciona a assistência para assim surgir mudanças para a melhoraria da qualidade do atendimento prestado para esse usuário (LOPES AS, et., 2015). Compreende-se que $\mathrm{o}$ acolhimento é uma prática que deve estar presente em todas as formas do cuidado, acolher o usuário auxilia na organização da demanda espontânea, assim ele se dá no momento em que o profissional compreende as necessidades do usuário e o direciona de acordo as suas necessidades, pode-se dizer que o acolhimento pode possibilitar a humanização que tem como prioridade a satisfação do usuário no atendimento constituindo uma garantia do acesso (BRASIL, 2013).

O acolhimento não deve ser restringido apenas na triagem para encaminhamento médico, ele visa humanizar as relações dos serviços de saúde e trabalhadores para garantia aos usuários de uma escuta qualificada (BRASIL, 2013). É importante compreender que o acolhimento não é restrito a médicos e enfermeiros, todos os profissionais da área da saúde devem estar aptos a acolher o usuário, sendo assim o acolhimento deve ser realizado pela equipe multiprofissional, ocorrendo durante todo o funcionamento do serviço, ou seja, não existe horário específico para que haja o acolhimento. Uma das grandes dificuldades é a compreensão dos profissionais sobre a implantação da humanização e acolhimento nos serviços de saúde preconizado pela PNH (NOBRE JPS, et al., 2016).

O SUS apesar dos avanços, ainda enfrenta alguns desafios a serem cumpridos, os profissionais não conseguem suprir com todas as expectativas do usuário, tendo como um dos fatores a falta de instrumentos e recursos de trabalho necessários que são preconizados, acrescenta-se ainda que o acolhimento é permeado por conflitos e contradições (CAMELO MS, et al., 2016). 
Mesmo após a implantação do acolhimento por meio da $\mathrm{PNH}$, ainda há práticas equivocadas existentes nos serviços de saúde, sendo necessários estudos que busquem refletir e conhecer essas práticas para possíveis mudanças, assim, o objetivo do presente estudo foi avaliar o nível de conhecimento e sistematização da produção científica vinculada à Política Nacional de Humanização em sua diretriz de acolhimento após sua implantação.

\section{MÉTODOS}

Para alcançar o objetivo do estudo foi realizado um estudo cienciométrico da literatura científica nacional e internacional acerca da Política Nacional de Humanização no que diz respeito ao acolhimento. A busca de artigos foi feita nas bases de dados indexados no United States National Library of Medicine (PubMed), e também no Scientific Eletronic Library Online (SciELO), disponível na Biblioteca Virtual em Saúde (BVS).

A pesquisa foi realizada entre setembro e outubro de 2020, foi utilizado o termo "acolhimento" em português e seu equivalente na língua inglesa, para a busca em inglês foi acrescentada a palavra Brasil. $\mathrm{Na}$ base de dados PubMed foi utilizado o filtro de dados Publication dates - 5 years e na base de dados SciELO foram designados os anos de 2016 a 2020.

Para a escolha e classificação dos artigos foi feita leitura dos títulos e resumos. Após esta etapa, os trabalhos escolhidos e incluídos no estudo foram classificados pelo ano de publicação, local de origem das publicações (baseado no primeiro autor), nome do periódico da publicação do artigo, e agrupados nos estratos WebQualis (A1, A2, B1, B2, B3, B4, B5 e C) na área das respectivas disciplinas. Os artigos que não tinham afinidade com a temática do acolhimento foram excluídos do estudo. A divisão WebQualis refere-se à estratificação da qualidade da produção científica utilizada pela Coordenação de Aperfeiçoamento de Pessoal de Nível Superior (CAPES) para avaliar os periódicos nas diferentes áreas de pesquisa no Brasil.

A classificação quanto às etapas referentes ao processo de implementação da Política Nacional de Humanização-PNH com a sua diretriz acolhimento foi um quesito para inclusão de estudos. Por conseguinte, os artigos foram analisados e categorizados de acordo com os seguintes setores de políticas públicas: Política HumanizaSUS e Política Nacional de Humanização (PNH). Finalmente, os dados foram tabulados e organizados em planilhas do programa Microsoft Excel 2016.

\section{RESULTADOS}

$\mathrm{Na}$ etapa inicial do processo de busca encontrou-se 586 publicações científicas, sendo 91 na base de dados PubMed e outras 495 na SciELO. Após a avaliação dos títulos e resumos foram excluídos os artigos duplicados e aqueles que não atenderam aos critérios de inclusão, resultando 67 artigos. Na análise dos anos de publicação artigos destacou-se o ano de 2014 que teve o maior número de publicações relacionado ao acolhimento (Figura 1).

Figura 1 - Número de artigos publicados no período entre 2016 a 2020 nas bases de dados secundários.

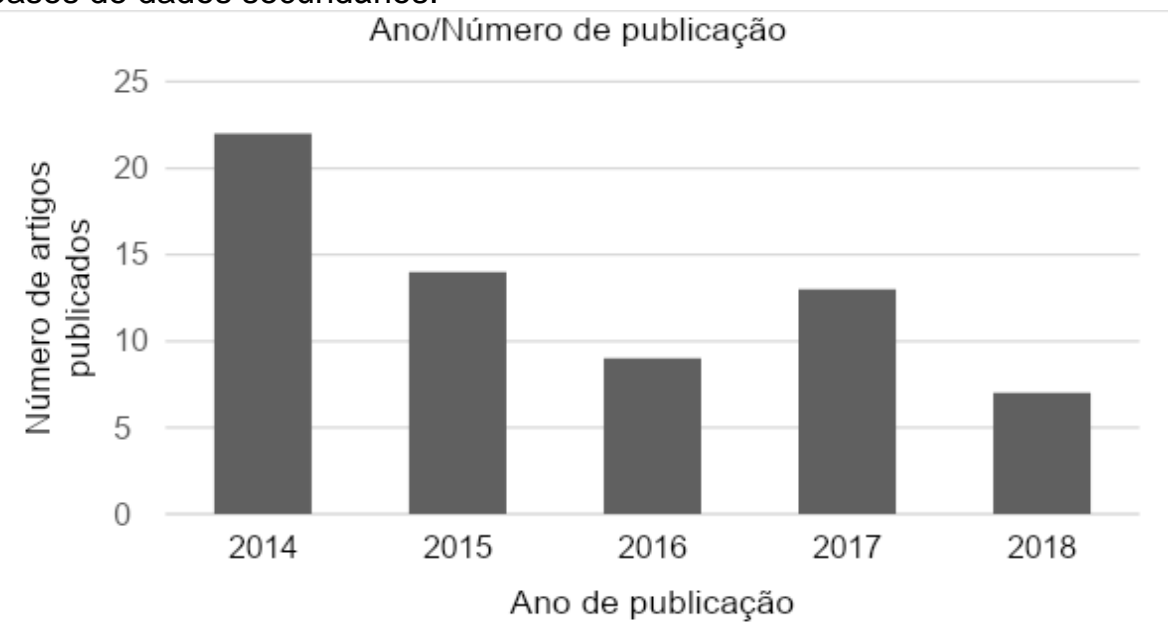

Fonte: Seixas ALM, et al., 2020. 
$\mathrm{Na}$ análise dos temas é possível identificar o maior quantitativo de estudos sobre o acolhimento na Unidade Básica de Saúde (UBS) e setores hospitalares, seguido de estudos que abordavam o acolhimento no Centro de Atenção Psicossocial (CAPS) (Figura 2).

Figura 2 - Número de artigos publicados no período entre 2016 a 2020 classificados conforme o cenário da pesquisa.

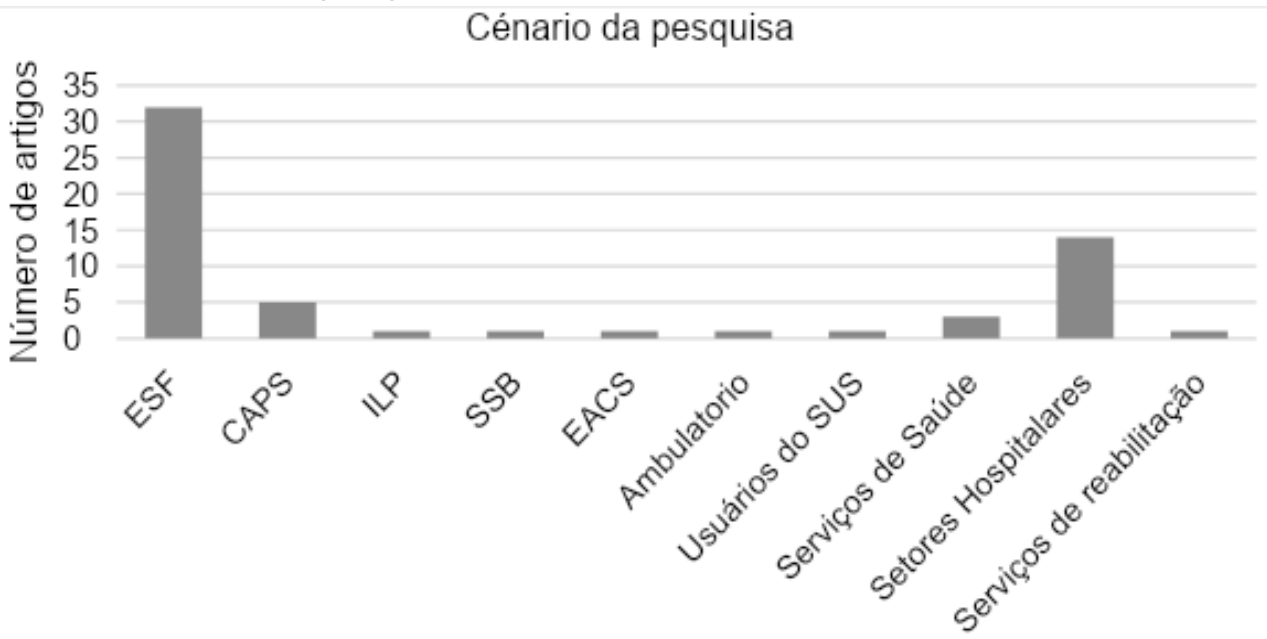

Eixos temáticos

Legenda: ESF: Estratégia Saúde da Família; CAPS: Centro de Atenção Psicossocial; ILP: Instituição de Terapia Intensiva; SSB: Serviço de saúde Bucal; EACS: Estratégia de Agente Comunitários de Saúde. Fonte: Seixas ALM, et al., 2020.

Em relação a classificação dos artigos quanto ao local de origem observou-se que os estados de São Paulo (14), Ceará (6), Pernambuco (5) e Minas Gerais (5) obtiveram 49\% dos 67 artigos relacionados ao acolhimento nos serviços de saúde. Em referência às publicações originárias de outros países não foram encontradas publicações que encaixassem nos critérios de inclusão estabelecidos (Figura 3).

Figura 3 - Número de artigos publicados no período entre 2016 a 2020 classificados quanto ao local de origem das pesquisas.

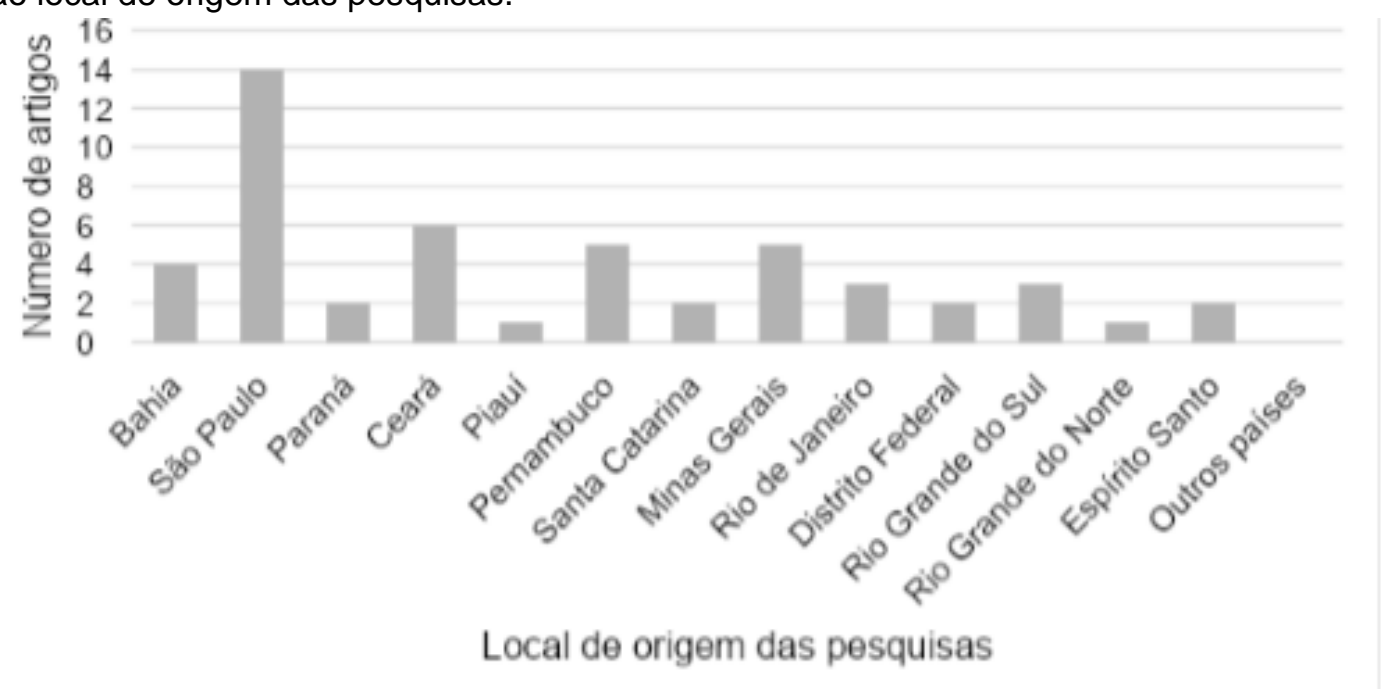

Fonte: Seixas ALM, et al., 2020. 
Quanto a classificação WebQualis analisados neste estudo evidenciou-se que 47,7\% dos artigos não são classificados nos estratos de Qualis, sendo A2 com 11,9\% e B1 com 32,8\% na área interdisciplinar. (Figura 4).

Figura 4 - Número de artigos publicados no período entre 2016 a 2020 classificados quanto a classificação WebQualis.

\section{Classificação de Qualis}

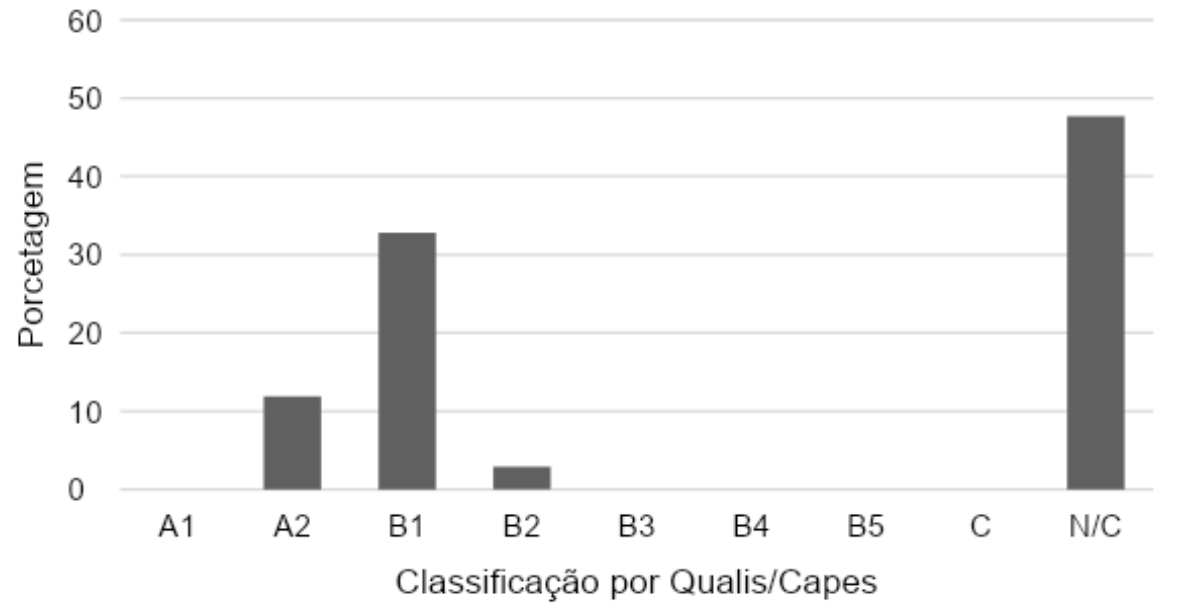

Legenda: N/C: Não classificados no WebQualis.

Fonte: Seixas ALM, et al., 2020.

\section{DISCUSSÃO}

Em relação a PNH após os dezessete anos de sua implementação considerando a sua diretriz de acolhimento, foi constatado que os as publicações tinham como cenário principal a Atenção Primária a Saúde, sendo que o foco das publicações não eram as práticas de acolhimento. Em 1988 foi criado o SUS, sendo firmados os princípios da universalidade, integralidade e equidade. A principal proposta desse sistema é a garantia de melhor qualidade de vida as pessoas, porém perante a esse princípio aproximaram-se alguns desafios a serem percorridos, sobretudo, no Brasil que é um país permeado de desigualdades socioeconômicas, tais desafios são basicamente a desvalorização dos trabalhadores e falta de recursos que acarretam precarização dos serviços de saúde, considerando todo esse cenário foi elaborada a Política Nacional de Humanização que visa humanizar as práticas de saúde e institucionalizar a valorização do profissionais, gestores e usuários (BRASIL, 2010).

A implantação da PNH em sua diretriz acolhimento nas práticas de saúde possibilita o aprofundamento de questões importantes vivenciadas nos serviços de saúde e a discussão sobre a relação entre profissionais e usuários que é extremamente importante por possibilitar a troca entre eles, promovendo o acolhimento das demandas do usuário, proporcionado um atendimento mais eficaz e proporcionando a reflexão de como está organizado o serviço de saúde em seu modelo assistencial, estimulando a repensar mudanças para mudar essas perspectivas (LOPES GVDO, et al., 2014).

A humanização referente ao atendimento no SUS pode ser compreendida como a inclusão e a valorização dos diferentes atores envolvidos no processo de atendimento e cuidado em serviços de saúde: usuários, trabalhadores, gestores e prestadores de serviços, para tanto este conceito está diretamente ligado à consolidação dos princípios de integralidade, universalidade e equidade no atendimento aos usuários (FIGUEIROA MN, et al., 2017).

De acordo com os resultados encontrados na presente investigação, a produção científica relacionada ao acolhimento mostrou-se limitada, o que inviabiliza discussões abrangentes. $O$ acolhimento no atendimento por possibilitar a humanização oferece a garantia do acesso e o atendimento de forma qualificada o que 
proporciona uma resposta efetiva do acesso ao usuário devido a resolutividade do seu problema ou encaminhamento ao serviço que solucione o mesmo.

O acolhimento não é padronizado, ou tem local e data marcada, este surge da demanda espontânea dos usuários. Não há necessidade de um profissional específico para realizá-lo. A implicação deste procedimento é a escuta do usuário, suas queixas e demandas, fazendo com que o cidadão atue como protagonista do seu processo saúde-doença e se responsabilize pela resolução com o apoio da rede de atenção (BRASIL, 2013).

Ainda que, em teoria, todos os profissionais de saúde sejam responsáveis por realizar o acolhimento, o que acontece, na maioria dos serviços de saúde, é que os profissionais de enfermagem são os principais envolvidos no processo do acolhimento. O que torna essa categoria profissional, como referência, no que diz respeito ao acolhimento e escuta qualificada dos usuários (SILVA et al., 2020).

O acolhimento pode ser reconhecido como uma via de mão dupla, uma vez que é um estágio do método de trabalho em que estabelece de que forma será feita a recepção de pessoas nos serviços, determinando assim como o serviço fará seu primeiro contato com os usuários, em um processo recíproco de reconhecimento, sendo que o usuário se identifica como cliente do serviço e, por conseguinte, o serviço o reconhece como um usuário com direitos estabelecidos relacionado ao serviço prestado, sendo visto também como uma tecnologia do método intermediário do trabalho em saúde que acontece em todos os ambientes em que ocorrem as interações entre usuário e trabalhadores (SILVA TF, et al., 2015).

Nessa discussão, a resolutividade é um dos quesitos da rede de atenção à saúde que tem a função de identificar demandas de saúde e vulnerabilidades, utilizando tecnologias distintas de cuidado individual e coletivo, mediante uma clínica ampliada capaz de realizar intervenções clínicas efetivas e construir vínculos positivos, com vistas para a ampliação dos níveis de autonomia dos indivíduos e coletividade (BRASIL, 2012).

Para haver eficácia no atendimento dos serviços de saúde é necessário que haja garantia da acessibilidade, resolutividade e universalidade, assim assumindo a função de acolher, é necessário que a equipe de saúde se reúna e discuta sobre como está sendo realizado o acolhimento dos usuários, qual o caminho que ele percorre até a resolutividade da sua procura ao sistema de saúde, quais são os profissionais que recepcionam este usuário, tornando indispensável uma auto avaliação de como está sendo realizado o processo para que assim ocorra uma mudança efetiva desse acesso, para transformar as práticas de saúde é necessário mudanças em seu processo de construção (BARROS MEB, et al., 2014).

As possibilidades para um acolhimento humanizado são muitas e o importante é que ocorra melhorias no atendimento do serviço de saúde, ocorrendo com a participação de toda a equipe que trabalha no serviço (BARROS MEB, et al., 2014). Alguns estudos mostram que há contradições entre as percepções e os relatos das experiências entre trabalhadores e gestores dos serviços de saúde como protagonistas do acolhimento. É notório o afastamento entre a teoria e a prática do acolhimento. Por um lado, o acolhimento é visto como um método de escuta qualificada e os vários tipos de acolher, em outro plano como retrata a prática, em muitas vezes ele limita-se de forma equivocada a estratégias para o atendimento da demanda espontânea a urgências (MOIMAZ SAS, et al., 2017).

Esse cenário deve ser transformado, pois a prática do acolhimento contribui na melhoria da (re)organização do serviço, visando ao atendimento da demanda espontânea nos serviços de saúde, sendo assim torna-se importante a implantação desse dispositivo de forma efetiva, pois ele possibilita o vínculo entre profissionais e usuários contribuindo também na qualidade do atendimento oferecido (CAMELO MS, et al., 2016). O acolhimento foi designado para colaborar com a qualidade dos sistemas de saúde possibilitando ao usuário acesso justo e integral no seu atendimento com a participação de multiprofissionais da saúde. Para se ter um atendimento acolhedor é necessário que a humanização ande em conjunto e entende-se que essa tarefa convoca todos os profissionais e gestores (SILVA IN, et al., 2018).

É importante qualificar o modo como está sendo desenvolvido o acolhimento, não devendo ser trabalhado de forma desarticulado e não tendo que ser comparado a uma atividade de triagem, é significativo salientar que o acolhimento deve ser realizado de acordo com a demanda espontânea em todos os momentos e não devendo ser definido um horário para que ocorra essa escuta (SILVA IN, et al., 2018). 
Assim, o acolhimento está presente na prestação de cuidado, nas reuniões entre usuários e trabalhadores de saúde, ao receber e ouvir os usuários e na construção de vínculos, mediante a escuta qualificada, evitando a desvalorização das queixas dos usuários e identificando demandas, sejam estas coletivas ou individuais e exercendo o papel de mecanismo de viabilização do acesso. Sendo assim, o serviço oferecido à população deve satisfazer às demandas dos usuários na sua excentricidade, assistindo o usuário no âmbito biopsicossocial (GOMIDE MFS, et al., 2018).

O acolhimento é uma ferramenta que irá fortalecer o vínculo entre os profissionais e usuários qualificando a assistência da saúde e humanizando as práticas, podendo contribuir para a compreensão dos usuários sobre as doenças e estimulando o autocuidado, dentre outros (BRASIL, 2017). Para garantir a atenção integral e humanizada é necessário que haja demonstração de comprometimento, interação de elos entre profissionais e usuários e sensibilidade pela equipe multidisciplinar de gestores e trabalhadores. Ainda é encontrado grandes desafios para se enfrentar para que possa efetivar a humanização nos serviços de saúde, umas delas é a ambiência, é preciso um ambiente harmonizado para que haja esse acolhimento humanizado (PELIOLI C, et al., 2014).

A PNH defende a preconização da importância da valorização da ambiência que é um auxiliador na organização do ambiente saudável e acolhedor. A ambiência interfere da forma em que será realizado esse processo de trabalho, sendo capaz de proporcionar espaços prazerosos de convivência coletiva (CLEMENTINO FS, et al., 2015). Partindo deste cenário, a humanização é vista como uma estratégia vasta e de natureza pública, que abrange a gestão dos serviços de saúde e a atenção prestada, colocando novamente em foco a problemática e desafios do SUS, no sentido de humanização da atenção e melhoria do processo de trabalho.

Para assumir de fato o acolhimento como uma diretriz deve haver um processo de mudanças de como é exercido essa estratégia de cuidado para que haja uma verdadeira transformação das práticas, devendo haver múltiplos aspectos técnicos e político-institucionais que contribuirá para a mobilização para atingir com êxito o acolhimento (BRASIL, 2013). O acolhimento nos serviços de saúde normalmente se confunde pela equipe como um cuidado que deve envolver apenas um profissional específico para exercer 0 ato de acolher, mas 0 acolhimento deve ser realizado por toda a equipe, não sendo restrito apenas para médicos e enfermeiros, ele deve ser visto como a necessidade de fazer uma escuta dinâmica e necessária (SILVA TF, et al., 2015).

É imprescindível ressaltar que o acolhimento deve ser uma ferramenta desenvolvida para fortalecer o vínculo entre profissionais e usuários, sendo preciso e importante aumentar os níveis de resolutividade e aperfeiçoar a organização dos serviços de saúde. Quando os profissionais entendem a função e a importância do acolhimento, estes tendem a respeitar os princípios e diretrizes do SUS para garantir a resolução das demandas dos usuários nos serviços de saúde (BARROS et al., 2018).

Com o objetivo de ser um dispositivo para atender o quesito de acesso, proporcionar vínculo entre população e equipe, usuário e profissional, o acolhimento visa discutir os processos de trabalho, estimular cuidado integral e alterar a clínica. Para tal, é necessário capacitar os profissionais para um bom atendimento desde a recepção até a resolução da demanda do paciente. Esse processo demanda métodos de cooperação mútua, que respeitem a constante negociação de conflitos na rotina dos serviços de saúde (SOUZA, et al., 2018).

Sendo considerável compreender que o acolhimento é visto de forma incorreta como norteador para o agendamento de consultas, sendo importante salientar que acolher a demanda não é direcionar todos os usuários a uma consulta médica, deve considerar então, o acolhimento como mecanismo que auxilia na diminuição da espera dos usuários que necessitam de atendimento. A qualidade do atendimento oferecido para esse usuário irá interferir diretamente no vínculo entre ele e os profissionais, uma boa escuta auxilia no melhor direcionamento das verdadeiras necessidades do usuário (GOMIDE MFS, et al., 2018).

Apesar a literatura mostrar que é imprescindível o acesso ao acolhimento na saúde, há lacunas de produções que abordam a influência de sua aplicação no cuidado contínuo e de forma educativa para os usuários. Sendo assim, torna-se importante compreender os fatores que influenciam a utilização dos serviços 
de saúde bem como as dificuldades encontradas pelos profissionais para suprir as demandas destes usuários (GIRÃO ALA e FREITAS CHA, 2016).

Uma das bases para o aperfeiçoamento do acolhimento é a capacitação dos profissionais e trabalhadores da saúde. A competência profissional, o domínio dos protocolos locais, bem como o domínio do conhecimento técnico, são bases para um acolhimento de qualidade frente às situações diárias de trabalho, entretanto, o que é indicado para o trabalhador exercer pode estar distante da realidade vivenciada por ele. Os princípios que norteiam os processos de trabalho devem ser continuamente remodelados por meio da utilização de saberes e práticas dos próprios trabalhadores e profissionais (WARMLING CM, et al., 2019).

Por fim, no sentido de reestruturação dos serviços de saúde, o acolhimento tem como exigência a gestão estratégica de pessoas, o que tem como resultado um canal definitivo de comunicação entre prestadores de serviços e usuário da mesma forma que na presteza da oferta de ações e serviços por meio de uma equipe comprometida profissionalmente com suas ações. Para a execução do acolhimento de qualidade, o apoio administrativo, a articulação entre serviço e programas assistenciais, o acesso ao sistema de informação, profissionais capacitados e pouca rotatividade profissional são subsídios que corroboram para uma boa prática (SOUZA TH, et al., 2016).

\section{CONSIDERAÇÕES FINAIS}

Após dezessete anos da implementação da PNH em relação a sua diretriz acolhimento, constatou-se que houve produções voltadas mais fortemente à implementação do acolhimento no cenário da Atenção Primária à Saúde, nesse sentido, houve uma produção limitada em relação às práticas de acolhimento como determina a PNH nos serviços de saúde, o que não permite o processo de avaliação de forma coletiva pelos usuários, gestores e profissionais de saúde. Esse estudo cienciométrico possibilitou compreender que no cenário de saúde nacional ainda há concepções equivocadas acerca do acolhimento, definindo-o como triagem, agendamento de consultas, consulta médica, pertencente a esse ou aquele profissional e com horário limitado para acontecer, dentre outros, e não vivenciando o acolhimento como formação de vínculo, compromisso e escuta qualificada. Sugere-se investigações sobre a temática a fim de esclarecer práticas inadequadas e formas de resolução, possibilitando avanços nas práticas de saúde no sistema de saúde brasileiro.

\section{REFERÊNCIAS}

1. BRASIL. Ministério da Saúde. Secretaria de Atenção à Saúde. Acolhimento à demanda espontânea. Brasília: Ministério da Saúde, 2013.

2. BRASIL. Ministério da Saúde. HumanizaSUS: Documentos base para gestores e trabalhadores do SUS. Brasília: Ministério da Saúde, 2010.

3. BRASIL. Ministério da Saúde. Política Nacional de Humanização e Gestão do SUS. A experiência da diretriz da Ambiência da Política Nacional de Humanização - PNH. Brasília: Ministério da Saúde, 2017.

4. BRASIL. Ministério da Saúde. Política Nacional de Atenção Básica. Brasília: Ministério da Saúde, 2012.

5. BARROS MEB, et al. O apoio institucional como dispositivo para implantação do acolhimento nos serviços de saúde. Interface. 2014; 18(1): 1107-1117.

6. BARROS MMAF, et al. Acolhimento em unidade de atenção primária à saúde: potencialidades e desafios. Revista de Saúde Pública SANARE. 2018; 17(2): 114-119.

7. BORGES JDM, SILVA LAA. O acolhimento na atenção básica à saúde: saberes e práticas. Rev enferm UFPE online. 2015; 9(5): 7887-7894.

8. CAMELO MS, et al. Acolhimento na Atenção primária à saúde na ótica de enfermagem. Acta Paul Enferm. 2016; 29(04): 463-468.

9. CLEMENTINO FS, et al. Acolhimento na Atenção Básica a partir da avaliação externa do programa de melhoria do acesso e da qualidade da Atenção Básica (PMAQ-AB). Revista Saúde e Ciência. 2015; 41(1): 62-80.

10. FIGUEIROA MN, et al. Acolhimento do usuário e classificação de risco em emergência obstétrica: avaliação da operacionalização em maternidade-escola. Escola Anna Nery. 2017; 21(4): 1-7.

11. GIRAO ALA, FREITAS CHA. Usuários hipertensos na atenção primária à saúde: acesso, vínculo e acolhimento à demanda espontânea. Rev. Gaúcha Enferm. 2016; 37(2): 1-7. 
12. GOMIDE MFS, et al. A satisfação do usuário com a atenção primária à saúde: uma análise do acesso e acolhimento. Interface - Comunicação, Saúde, Educação. 2018; 22(65): 387-398.

13. LOPES AS, et al. O acolhimento na Atenção Básica em Saúde: relação de reciprocidade entre trabalhadores e usuários. Saúde em Debate. 2015; 39(204): 114-123.

14. LOPES GVDO, et al. Acolhimento: quando o usuário bate à porta. Rev Bras Enferm. 2014; 67(1): 104-110.

15. MARTINS CP, LUZIO CA. Política Humaniza SUS: ancorar um navio no espaço. Interface. 2017; $21(60)$ : 13-22.

16. MARTINS CP. A política Nacional de Humanização na produção de interflexões no modelo hegemônico de cuidar e gerir no SUS: habitar um paradoxo. Tese (Doutorado em Psicologia) - Faculdade de Ciências e Letras de Assis. Universidade Estadual Paulista, São Paulo, 2015; 192 p.

17. MOIMAZ SAS, et al. Qualificação do acolhimento nos serviços de saúde bucal. Cad. saúde colet. $2017 ; 25$ (1): 1-6.

18. NOBRE JPS, et al. A percepção dos trabalhadores de saúde sobre o acolhimento no contexto da Atenção Básica. Revista Expressão Católica. 2016; 1(1): 13-23.

19. PELIOLI C, et al. O acolhimento em saúde: uma revisão sistemática em periódico brasileiro. Estudos de Psicologia. 2014; $31(2): 225-235$.

20. SILVA IN, PEREIRA VA, ARAÚJO LCM. Implantação da política Nacional de Humanização (PNH): conquistas e desafios para a assistência em saúde. Gepl News. 2018; 1(1): 1-7.

21. SILVA TF, ROMANA VF. Sobre o acolhimento: discurso e prática em Unidades Básicas de Saúde do município do Rio de Janeiro. Saúde Debate. 2015; 39(105): 363-374.

22. SOUZA ECF, et al. Acesso e acolhimento na atenção básica: uma análise da percepção dos usuários e profissionais de saúde. Cad. Saúde Pública. 2018; 24(1): 100-110.

23. SOUZA TH, et al. Recepção: ponto estratégico para o acesso do usuário ao sistema único de saúde. Texto contexto - enferm. 2016; 25(3): 1-8.

24. WARMLING CM, et al. Acolhimento e acesso de necessidades de saúde bucal e o agir profissional na Atenção Primária à Saúde. Interface - Comunicação, Saúde, Educação. 2019; 23(1): 1-15.

25. SILVA TCS, et al. Acolhimento noturno em um Centro de Atenção Psicossocial III. Rev. Bras. Enferm. 2020; 73(1): 17. 